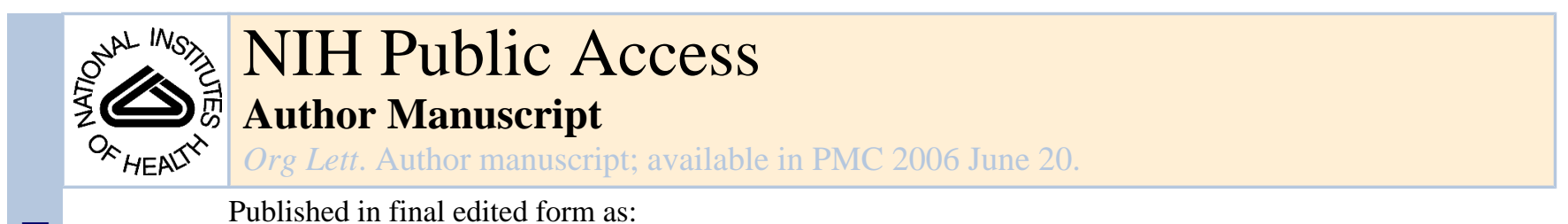

Org Lett. 2005 May 26; 7(11): 2153-2156.

\title{
Synthetic Studies on the Bryostatins: Synthetic Routes to Analogues Containing the Tricyclic Macrolactone Core
}

\author{
Gary E. Keck and Anh P. Truong \\ Department of Chemistry, University of Utah, 315 South 1400 East RM 2020, Salt Lake City, Utah \\ 84112-0850keck@chemistry.utah.edu
}

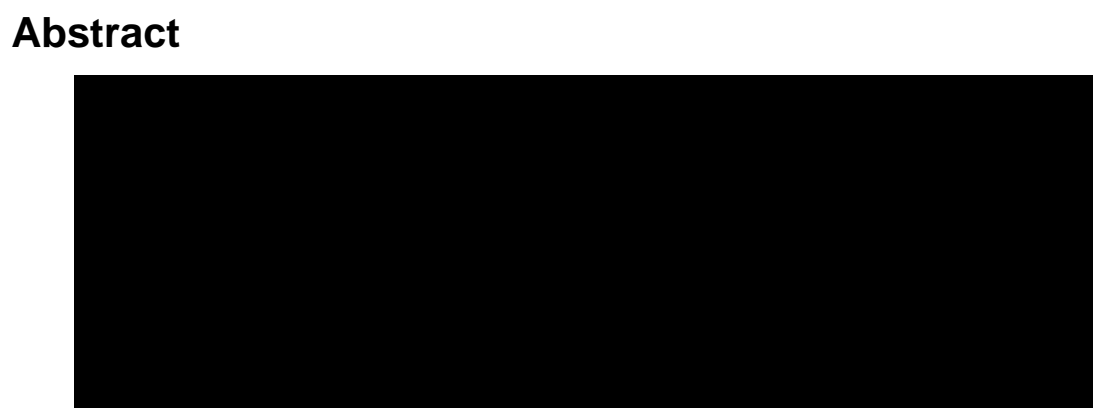

Synthesis of the first of a projected series of bryostatin analogues has been accomplished in 26 steps and $2.2 \%$ overall yield. In this letter, we detail two approaches to the structural core of these tricyclic macrolactone bryostatin analogues. The key features of the route include BITIP-catalyzed asymmetric allylation reactions and Mukaiyama aldol reactions, a chelation-controlled allylation, pyran annulation reactions, and macrolactonization.

\begin{abstract}
In the previous paper, we described the synthesis of a potential $\mathrm{C}_{9}-\mathrm{C}_{27}$ intermediate for a projected synthesis of bryostatin $1 .{ }^{1}$ However, at this point we elected to delay our efforts on the synthesis of bryostatin 1 itself in favor of investigations of the synthesis of truncated analogues using the same basic overall strategies. Biological evaluation of bryostatin analogues has shown very encouraging results. ${ }^{2}$ In particular, the efforts of Wender and co-workers in the bryostatin analogue area have highlighted what can be achieved in the area of simplified analogue design. Several interesting facets from Wender's studies have also shed light on the structure-activity relationships of these compounds. ${ }^{3}$ It was our hope that the syntheses of bryostatin analogues would not only serve as highly advanced "model" studies for the synthesis of bryostatin 1 itself but also give access to structurally simplified analogues of the bryostatins that retained or exceeded the levels of activity exhibited by the natural materials. Synthetic access to such structures and the attendant tuning of structure could in turn provide a better understanding of the mechanism of action of these agents and better define structure-activity relationships in this area.

In the previous article, we demonstrated that a C-ring enal could be sucessfully coupled with a simple hydroxyallylsilane using the pyran annulation reaction to incorporate the B-ring carbons in one synthetic step. However, as we gained more experience with this reaction, it became clear that it was a particulary robust and high-yielding process that might be implemented in a more strategic role. In particular, we were intrigued by the possibility of using this process to bring together subunits corresponding to rings $\mathrm{A}$ and $\mathrm{C}$ with concomitant
\end{abstract}

Supporting Information Available: Spectral $\left({ }^{1} \mathrm{H}\right.$ and ${ }^{13} \mathrm{C}$ NMR) data for materials described herein. This material is available free of charge via the Internet at http://pubs.acs.org. 
formation of the B-ring, according to the strategy shown in Scheme 1. As indicated, either the A- or C-ring intermediates could play the role of the hydroxy allylsilane. In addition, the same approach could be applied in the service of analogue synthesis, which would serve as an advanced model study for the synthesis of bryostatin 1 itself. We describe herein the implementation of this strategy for the synthesis of the tricyclic macrolactone core of the bryostatins.

The dissection in which ring $\mathrm{C}$ played the role of the hydroxy allylsilane component was examined first. In the preceding paper, we described the preparation of the $\mathrm{C}$-ring enal intermediate 5. To obtain the $\beta$ - hydroxy allylsilane, our plan was to utilize our catalytic asymmetric allylation (CAA) reaction with this enal. ${ }^{4}$ However, the execution of this simple step was complicated due to the presence of sensitive functionalities on the C-ring. We were unable to obtain an acceptable yield using a catalytic amount of the BITIP catalyst with this enal and the 2-(trimethylsilylmethyl) allylstannane reagent $\mathbf{6}$. However, success was obtained using a large excess of the BITIP reagent. The product 7 was obtained in modest $(67 \%)$ yield due to some decomposition during the reaction.

The reaction partner needed for this annulative approach to fragment coupling is an aldehyde incorporating the A-ring pyran. As indicated in Scheme 3, we envisioned that the A-ring pyran $\mathbf{8}$ could itself be accessed by a pyran annulation between the simple hydroxy allylsilane $\mathbf{9}$ and aldehyde 10. We chose to use a thiol ester as the precursor to the carboxylate at $\mathrm{C}_{1}$ since we anticipated that it could be selectively hydrolyzed under mild and specific conditions to liberate the carboxylic acid needed for esterification with the $\mathrm{C}_{26}$ hydroxyl group of the C-ring fragment. In addition, using this approach, the catalytic asymmetric Mukaiyama aldol reaction using BITIP catalysis would provide a very convenient means to set the $\mathrm{C}_{3}$ stereocenter. ${ }^{5}$

The synthesis of the aldehyde component, 10, began with the BITIP-catalyzed Mukaiyama aldol reaction of the PMB-protected $\beta$-hydroxy aldehyde 11 with thiosilyl ketene acetal 12. The resulting alcohol was protected as the TBS ether to give 13 in $60 \%$ overall yield and in $95 \%$ ee. The PMB ether was then removed under standard conditions using DDQ in a methylene chloride-water mixture. Immediate oxidation of the crude alcohol so produced with PCC afforded the desired aldehyde $\mathbf{1 0}$ in $76 \%$ yield from the PMB ether $\mathbf{1 1}$. Thus, aldehyde 10 was obtained in four simple steps from readily available starting materials (Scheme 4).

The hydroxy allylsilane 9 was prepared using stannane 6 and $(R)$-BITIP catalyst to set the stereocenter at $\mathrm{C}_{9}$. This reaction proceeded in good yield $(81 \%)$ and excellent selectivity $(95 \%$ ee) on a 3-5 g scale.

These two fragments were then coupled via the pyran annulation reaction to give the desired pyran 14 in excellent (91\% isolated) yield. ${ }^{6}$ Fortunately, this reaction went smoothly, despite the possibility of potentially complicating side reactions such as $\beta$-elimination or cleavage of the tert-butyl thiol ester. Selective deprotection of the BPS group and oxidation of the resulting alcohol then afforded the desired A-ring aldehyde $\mathbf{8}$ in good yield. As shown in Scheme 4, the synthesis of the A-ring aldehyde was completed in seven steps and in $31 \%$ overall yield.

\section{Coupling of the $\mathrm{C}_{1}-\mathrm{C}_{11}$ and $\mathrm{C}_{12}-\mathrm{C}_{27}$ Fragments Using the Pyran Annulation Reaction}

With the synthesis of the A-ring aldehyde $\mathbf{8}$ accomplished, we turned our attention to the coupling of these fragments by reacting C-ring hydroxy silane 7 with A-ring aldehyde 8 in the presence of TMS triflate. We were pleased to find that the annulation reaction gave us the desired product 15 in good yield (63\% isolated yield) despite the presence of potentially problematic acid-sensitive functionality such as the enol ether of the C-ring pyran (Scheme 5). 


\section{Sequential Annulation Approach to the Bryostatin Core}

Although this synthesis of the tricyclic core of bryostatin analogues is flexible, we were concerned about the use of excess chiral promotor in the sequence of reactions leading to 15 . Thus, we decided to investigate an alternative strategy for the synthesis of the tricycle 15. In this alternative approach, the tricyclic material was envisioned to arise from two sequential annulation reactions starting from the enal $\mathbf{5}$. The first would use the same hydroxy allylsilane as in our previous report; ${ }^{1}$ the second would use a more complex hydroxy allylsilane component to bring in carbons $1-8$ with the required stereocenters in this fragment already established. Thus, we would use the same BC-ring intermediate prepared earlier ${ }^{1}$ in a second annulation with the more complex $\beta$-hydroxy allylsilane $\mathbf{1 9}$.

The synthesis of the $\mathrm{C}_{1}-\mathrm{C}_{8}$ fragment began with readily available aldehyde $\mathbf{1 6}$ (Scheme 6). A catalytic asymmetric allylation (CAA) reaction using allyltri- $n$-butylstannane and our normal CAA conditions afforded the homoallylic alcohol in excellent yield and with excellent selectivity $(90 \%, 96 \%$ ee). Protection of this alcohol as the PMB ether was best accomplished using PMBBr and KHMDS to give 17. Oxidative cleavage of the alkene then gave the desired PMB-aldehyde 18 in good yield (80\% over two steps).

Incorporation of the allylsilane in this instance was accomplished with the desired stereochemical result at $\mathrm{C}_{5}$ by simply using a chelation-controlled addition with 1,3asymmetric induction; asymmetric catalysis was unnecessary. Thus, as expected, ${ }^{8}$ reaction of the $\beta$-OPMB-aldehyde 18 with silyl stannane 6 using $\mathrm{MgBr}_{2} \cdot \mathrm{Et}_{2} \mathrm{Oat}-78{ }^{\circ} \mathrm{C}$ proceeded with complete stereoselectivity at $\mathrm{C}_{5}$, and in quantitative yield (Scheme 6). With the desired $\beta$ hydroxy allylsilane 19 in hand, the pyran annulation event was found to occur smoothly. Reaction of this silane with aldehyde 20 and TMS triflate, in ether at $-78{ }^{\circ} \mathrm{C}$, afforded the desired tricyclic $\mathrm{C}_{1}-\mathrm{C}_{27}$ subunit $\mathbf{2 1}$ in good yield (80\%) and with all requisite stereocenters now in place (Scheme 6). ${ }^{9}$

\section{Elaboration of the ABC Tricyclic Core}

The C-ring glycal was elaborated first. Treatment of the $\mathrm{ABC}$ tricycle $\mathbf{2 1}$ with $m$-CPBA in $\mathrm{MeOH},{ }^{10}$ followed by oxidation with TPAP-NMO, afforded the desired ketone at $\mathrm{C}_{20}$. It is noteworthy that considerable experimentation was required to determine a suitable order of functional group manipulations en route to the seco-acid. We first deprotected the PMB ethers at $\mathrm{C}_{3}$ and $\mathrm{C}_{25}$, followed by the deprotection of the BPS ether at $\mathrm{C}_{1}$. Many attempts to selectively oxidize the primary $\mathrm{C}_{1}$ hydroxyl in the presence of the free secondary hydroxyls at $\mathrm{C}_{3}$ and $\mathrm{C}_{25}$ using $\mathrm{PhI}(\mathrm{OAc})_{2}$ and TEMPO gave only a trace of the desired product and mainly unidentified decomposition products. A successful sequence elaborated $\mathrm{C}_{1}$ to the acid prior to removal of the PMB ethers. Thus, the BPS ether at $C_{1}$ was removed first, by reaction with TBAF. The resulting diol 22 was then oxidized to keto-aldehyde $\mathbf{2 3}$ using TPAP-NMO. This material was then subjected to a Pinnick oxidation ${ }^{11}$ to give the carboxylic acid. Finally, the PMB ether protecting groups at $\mathrm{C}_{3}$ and $\mathrm{C}_{25}$ were removed to give the desired dihydroxy acid 24 in good yield (80\%).

Having successfully overcome this particular hurdle, we then faced the critical issue of macrolactonization of the seco-acid in the presence of the hydroxyls at both $\mathrm{C}_{3}$ and $\mathrm{C}_{25}$ and also the removal of the Me and BOM ethers at $\mathrm{C}_{19}$ and $\mathrm{C}_{26}$. Macrolactonization was achieved using a modified Yamaguchi mixed anhydride esterification protocol to give the desired 20membered lactone 25. ${ }^{12}$ Finally, the BOM group and methyl ether at $\mathrm{C}_{26}$ and $\mathrm{C}_{19}$ were removed under very mild and specific conditions $\left(\mathrm{LiBF}_{4}\right.$, aqueous $\left.\mathrm{CH}_{3} \mathrm{CN} / \mathrm{H}_{2} \mathrm{O}\right)$ to give the dihydroxy macrolactone 26 in $62 \%$ yield. ${ }^{13}$ 
The synthesis of $\mathbf{2 6}$ requires 26 steps in total and has a longest linear sequence of 23 steps, considerably less arduous than required for the synthesis of a natural bryostatin. Efforts to elaborate this structure to more closely resemble the naturally occurring structures, as well as to provide biological evaluation of these agents, are in progress and will be reported in due course.

\section{Supplementary Material}

Refer to Web version on PubMed Central for supplementary material.

\section{Acknowledgment}

Financial assistance provided by the National Institutes of Health (through Grant GM-28961) is gratefully acknowledged.

\section{References}

1. Keck GE, Truong AP. Org. Lett 2005;7:2149. [PubMed: 15901156]

2. (a) Hale KJ, Hummersone MG, Manaviazar S, Frigerio M. Nat. Prod. Rep 2002;19:413. [PubMed: 12195811] and references therein (b) Hale KJ, Frigerio M, Hummersone MG, Manaviazar S. Org. Lett 2003;5:503. [PubMed: 12583754]

3. Wender PA, Baryza JL, Bennett CE, Bi FC, Brenner SE, Clarke MO, Horan JC, Kan C, Lacote E, Lippa B, Nell PG, Turner TM. J. Am. Chem. Soc 2002;124:13648. [PubMed: 12431074] (b) Wender PA, De Brabander J, Harran PG, Jimenez J-M, Koehler MFT, Lippa B, Park C-M, Shiozaki M. J. Am. Chem. Soc 1998;120:4534. (c) Wender PA, Hinkle KW, Koehler MFT, Lippa B. Med. Res. Rev 1999;19:388. [PubMed: 10502742] (d) Wender PA, Lippa B. Tetrahedron Lett 2000;41:1007. (e) Wender PA, Mayweg AVW, VanDeusen CL. Org. Lett 2003;5:277. [PubMed: 12556171]

4. (a) Keck GE, Li X-Y, Krishnamurthy D. J. Org. Chem 1995;60:5998. (b) Keck GE, Krishnamurthy D. Synth. Commun 1996;26:367. (c) Keck GE, Krishnamurthy D. Org. Synth 1998;75:12. (d) Keck GE, Abbott DE, Boden EP, Enholm EJ. Tetrahedron Lett 1983;25:3927.

5. Keck GE, Krishnamurthy D. J. Am. Chem. Soc 1995;117:2363.

6. Keck GE, Covel JA, Schiff T, Yu T. Org. Lett 2002;4:1189. [PubMed: 11922815]

7. This synthetic plan is quite flexible since the annulation can be approached from two different directions to form the B-ring pyran. In fact, we were able to demonstrate that the pyran annulation reaction worked equally well with the roles of the annulation partners reversed.

8. (a) Keck GE, Castellino S, Wiley MR. J. Org. Chem 1986;5:5478. (b) Evans DA, Dart MJ, Duffy JL, Yang MG, Livingston AB. J. Am. Chem. Soc 1995;117:6619. (c) Evans DA, Dart MJ, Duffy JL, Yang MG. J. Am. Chem. Soc 1996;118:4322.

9. Aldehyde $\mathbf{2 0}$ was prepared from the corresponding BPS ether by deprotection using TBAF followed by oxidation with TPAP-NMO. For the synthesis of this BPS ether, see ref ${ }^{1}$.

10. Evans DA, Carter PH, Charette AB, Prunet JA, Lautens M. J. Am. Chem. Soc 1999;121:7540.

11. Bal BS, Childers WE Jr. Pinnick HW. Tetrahedron 1981;37:2091.

12. Inanaga J, Hirata K, Saeki H, Katsuki T, Yamaguchi M. Bull. Chem. Soc. Jpn 1979;52:1989.

13. Zakarian A, Batch A, Holton RA. J. Am. Chem. Soc 2003;125:7822. [PubMed: 12822999] 


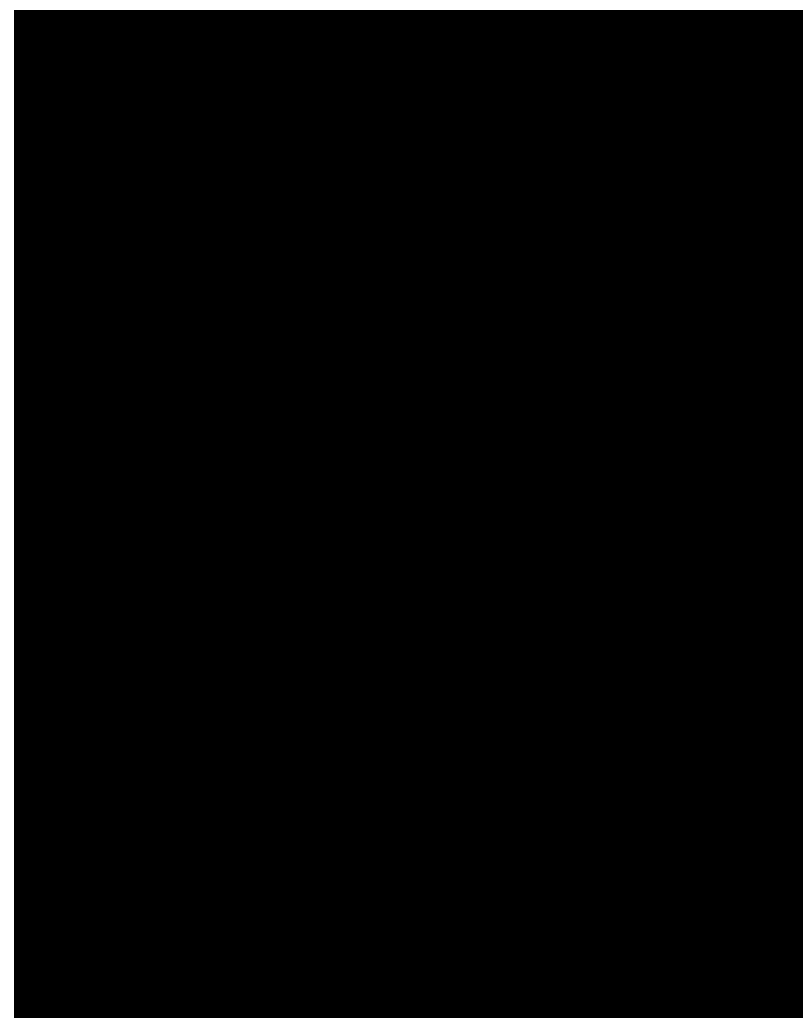

Scheme 1.

Retrosynthetic Analysis of the Bryostatins 


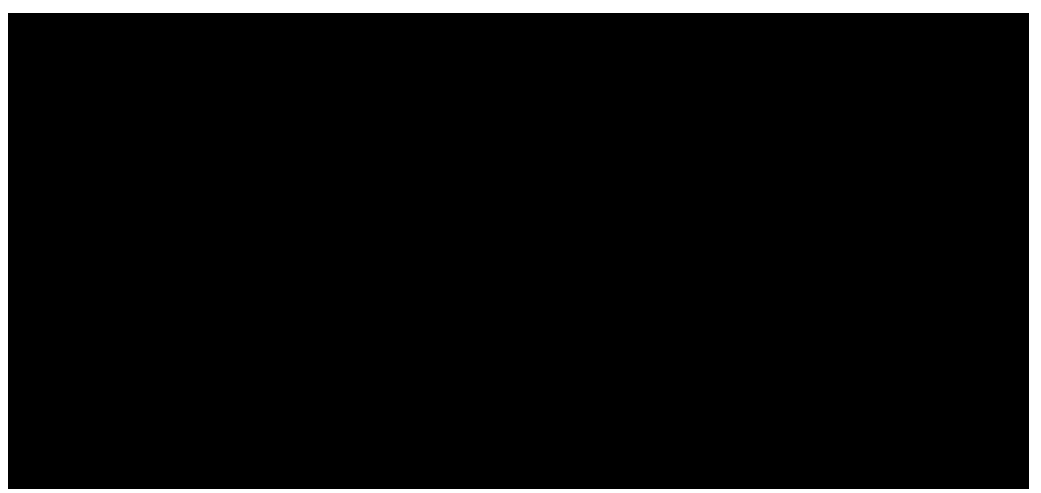

Scheme 2.

Synthesis of the $\mathrm{C}_{12}-\mathrm{C}_{27}$ Fragment 


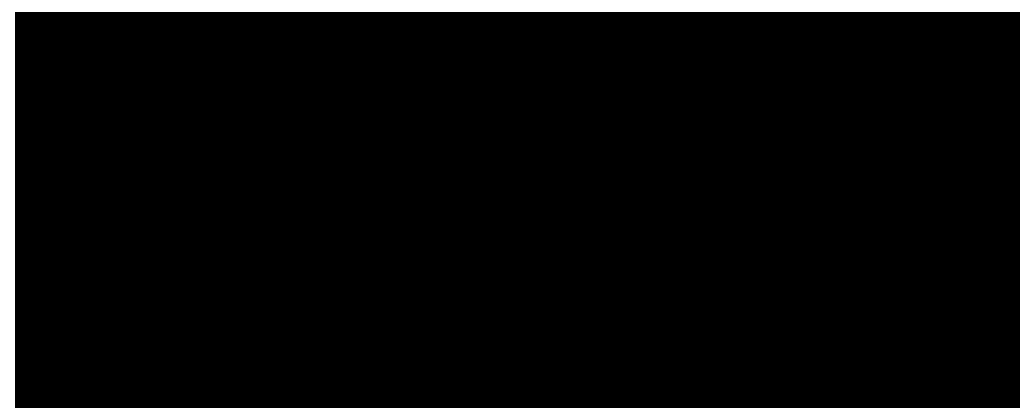

Scheme 3.

Retrosynthesis of the A-Ring Aldehyde 


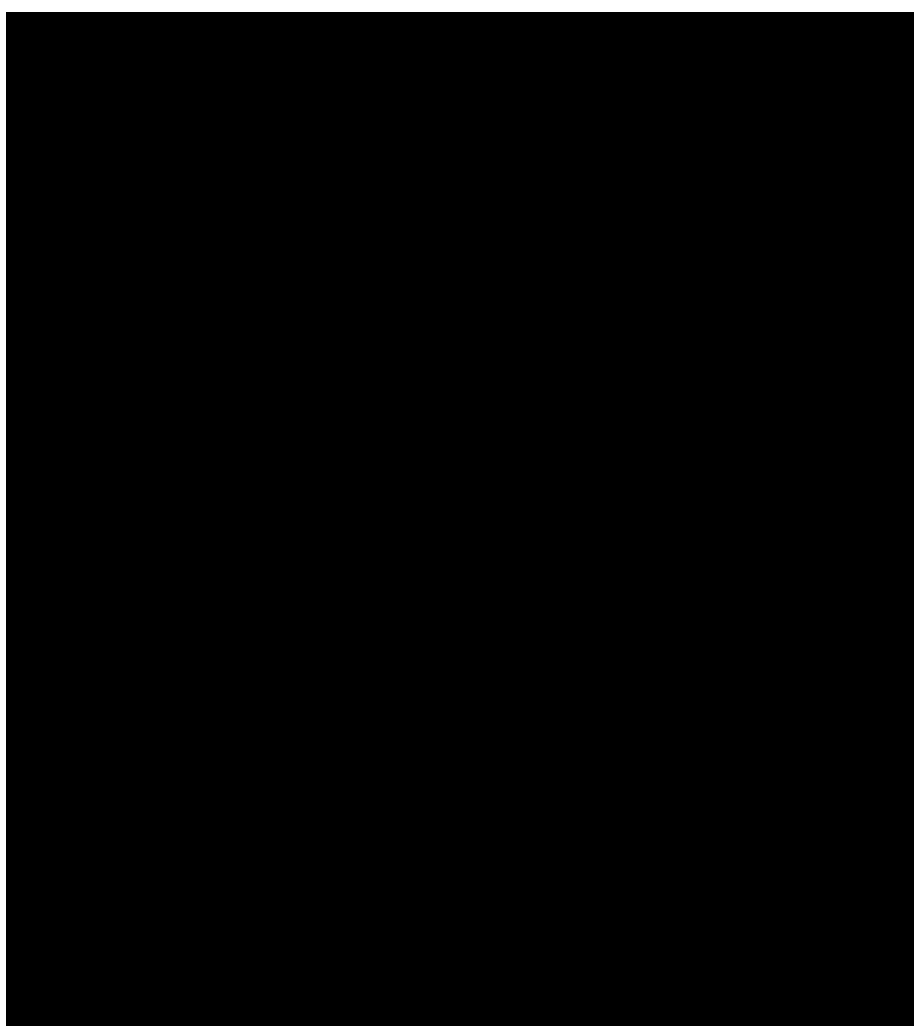

Scheme 4.

Synthesis of the A-Ring Pyran-aldehyde 


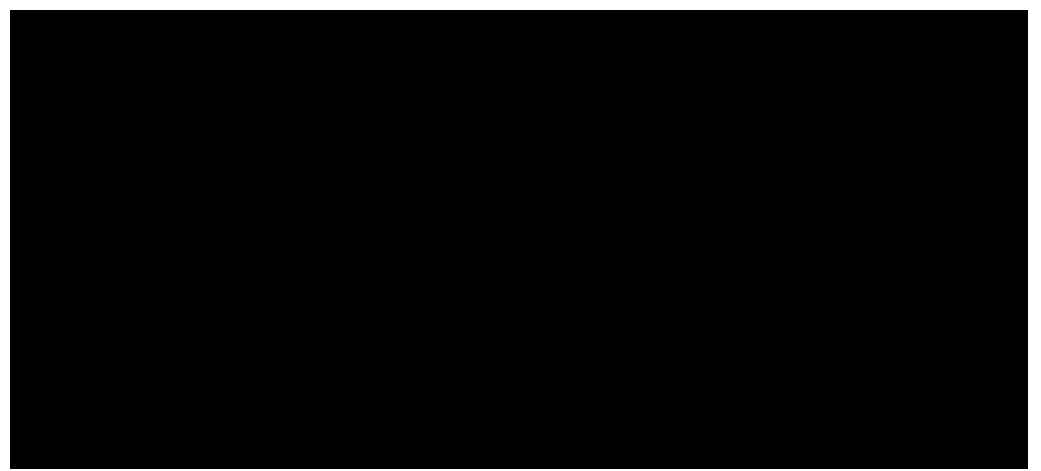

Scheme 5.

Union of Rings A and C via Pyran Annulation 
Preparation of the $\mathrm{C}_{1}-\mathrm{C}_{8}$ Fragment
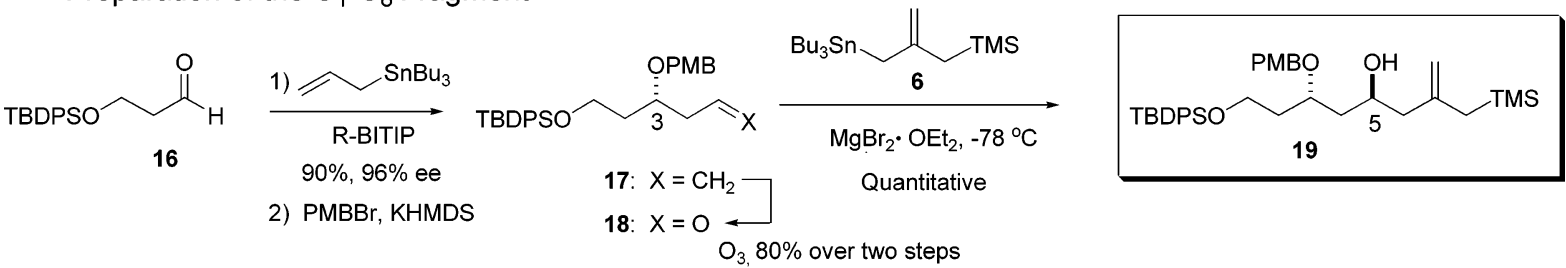

Elaboration of the $A B C$ tricyclic core
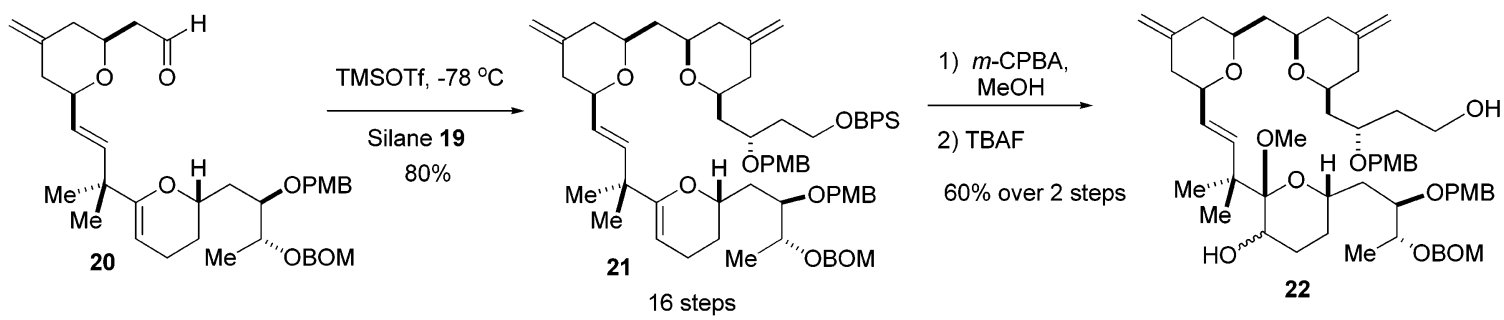

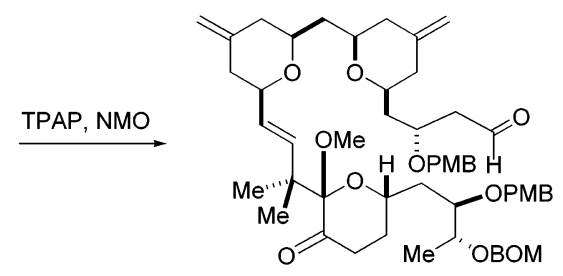

23

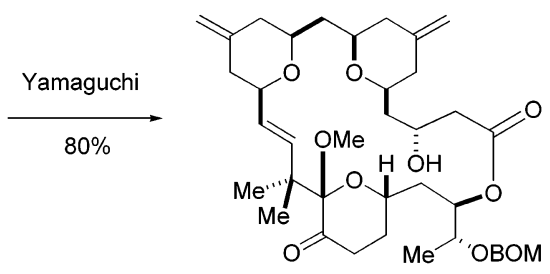

25

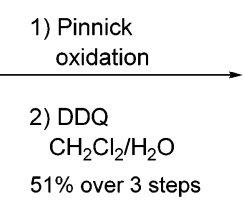

$51 \%$ over 3 steps
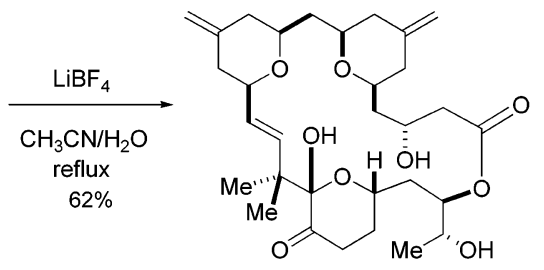

26

Scheme 6.

Completion of the Bryostatin Core Structure 